\title{
Optimizing experimental parameters for the projection requirement in HAADF-STEM tomography
}

\author{
R. Aveyard ${ }^{\mathrm{a}, *}$, Z. Zhong $^{\mathrm{b}}$, K.J. Batenburg ${ }^{\mathrm{b}}$, B. Rieger ${ }^{\mathrm{a}}$ \\ a Department of Imaging Physics, Delft University of Technology, 2628CJ Delft, The Netherlands \\ ${ }^{\mathrm{b}}$ Centrum Wiskunde and Informatica, Science Park 123, NL-1098 XG Amsterdam, The Netherlands
}

\section{A R T I C L E I N F O}

\section{Article history:}

Received 4 November 2016

Revised 22 February 2017

Accepted 5 March 2017

Available online 7 March 2017

\section{Keywords:}

HAADF

STEM

Simulation

Tomography

Projection requirement

\begin{abstract}
A B S T R A C T
Tomographic reconstruction algorithms offer a means by which a tilt-series of transmission images can be combined to yield a three dimensional model of the specimen. Conventional reconstruction algorithms assume that the measured signal is a linear projection of some property, typically the density, of the material. Here we report the use of multislice simulations to investigate the extent to which this assumption is met in HAADF-STEM imaging. The use of simulations allows for a systematic survey of a range of materials and microscope parameters to inform optimal experimental design. Using this approach it is demonstrated that the imaging of amorphous materials is in good agreement with the projection assumption in most cases. Images of crystalline specimens taken along zone-axes are found to be poorly suited for conventional linear reconstruction algorithms due to channelling effects which produce enhanced intensities compared with off-axis images, and poor compliance with the projection requirement. Off-axis images are found to be suitable for reconstruction, though they do not strictly meet the linearity requirement in most cases. It is demonstrated that microscope parameters can be selected to yield improved compliance with the projection requirement.
\end{abstract}

(c) 2017 Elsevier B.V. All rights reserved.

\section{Introduction}

In scanning transmission electron microscopy (STEM) a focussed electron beam is directed through a small part of the specimen, and some part of the intensity distribution is measured in the back focal plane. A high angle annular dark field (HAADF) detector is designed to measure the part of the beam that is incoherently scattered by Coulomb interactions with nuclei in the specimen. The measured signal is the sum of the high-angle scattering contributions of all nuclei in the path of the beam. As atoms with heavier nuclei present larger scattering cross-sections, the signal should be proportional to the thickness of the specimen beneath the incident beam position, and some power of the atomic number of the constituent nuclei. By raster scanning the beam across the specimen, a grid of pixels is generated, each with a value representing the mass-thickness of the specimen beneath the corresponding incident position. If the specimen is incrementally tilted and re-scanned, a dataset is generated that can be mathematically processed by a tomographic reconstruction algorithm to yield a grid of $3 \mathrm{D}$ voxels with values representing the scattering contribution

\footnotetext{
* Corresponding author.

E-mail addresses: r.a.aveyard@tudelft.nl (R. Aveyard), b.rieger@tudelft.nl (B. Rieger).
}

from that volume. This can be interpreted to yield a 3D model of the specimen. The implementation of this type of STEM tomography has been reported in a number of publications [1-3].

Conventional back projection tomographic reconstructions of HAADF images implicitly assume that the signal measured at each incident beam position is linearly proportional to the massthickness of the specimen beneath it. This is known as the projection criterion. The scattering mechanism that produces the HAADFSTEM signal necessarily modifies the beam as it progresses through the specimen, so the beam reaching a voxel at the bottom of the specimen is different from that at the top. The scattering contribution from a voxel is not just dependent on the nuclei in that voxel, but also of the form of the beam reaching the voxel. Consequently, the signal in HAADF-STEM does not vary linearly with mass-thickness, as has been observed in a number of cases [4-9].

Fortunately, the projection criterion can be relaxed to a requirement that the relationship between the signal and the massthickness of the specimen need only be monotonic, if the deviation from linearity is known, so that it can be corrected for in postprocessing [10]. Unfortunately, the relationship between the signal and mass-thickness, hereafter referred to as the projection relation, is usually not known. The non-linearity is often not corrected for during reconstruction and some inaccuracy is incurred. A procedure to provide a correction has been proposed by Van den Broek 
et al. [5]. This procedure involves calibrating the HAADF detector so that the signal measured at all specimen orientations can be expressed as a fraction of the total beam intensity. The HAADF signal is assumed to complement a bright field signal which decays with mass-thickness in accordance with a Beer-Lambert-like attenuation. The applicability of this correction is limited to specimens with low mass-thicknesses for which the occurrence of multiple scattering, and scattering beyond the outer range of the HAADF detector, can be considered negligible [5].

In this work, the extent to which HAADF-STEM produces nonlinear projection relations is investigated by means of multislice simulations. A range of microscope parameters and specimen materials are considered, in order to inform optimal experimental design to best approximate linear projection. In the coming section, we introduce the simulation procedure used to produce the data in this report, as well as the structural properties of the example specimens used. In the subsequent sections, the projection relations of a range of amorphous and crystalline materials are demonstrated for different microscope settings. Whilst it is impossible to conduct a comprehensive survey, the results reported here offer valuable insights for experimental design and give an indication of the extent to which deleterious non-linearities occur in HAADFSTEM imaging.

\section{Methods}

The linearity of HAADF-STEM can be investigated experimentally using wedge shaped samples [11], however, simulations allow for a survey of a broad range of specimens, which are perfectly defined, in ideal imaging conditions, and for a range of microscope parameters. In addition, multislice simulations facilitate the inspection of the angular distribution of the electron intensity at any z-slice depth in the specimen, allowing the projection relation to be recorded during the propagation of electron beams through a thick specimen. All the simulations described in this work were performed using a multislice program developed previously by the authors Aveyard and Rieger [12]. The specimen in each simulation consists of a $\sim 10 \times 10 \times 40 \mathrm{~nm}$ column elongated along the optical axis. For computational purposes, the specimen is placed in a $\sim 17 \times 17 \times 40 \mathrm{~nm}$ supercell, sampled by a series of $7000 \times$ 7000 pixel arrays transverse to the optical axis. With a pixel size of $2.4 \mathrm{pm}$, the simulation is bandwidth limited to an angular range of 270 mrad at $300 \mathrm{kV}$. In all the simulations the electron wavefunction is aberration-free with the beam focussed on the uppermost surface of the specimen. The beam is described by a $4096 \times 4096$ pixel array with real space dimensions of $10 \times 10 \mathrm{~nm}$. The broadest beam in the focal plane that is used in this work is generated at an accelerating voltage of $100 \mathrm{kV}$, and a convergence semi-angle of $10 \mathrm{mrad}$, and has a full width at half maximum of $2.6 \AA$. The imaging region is a $10 \times 10 \AA$ square in the centre of the column, to give an $4.5 \mathrm{~nm}$ specimen border around the imaging region, to ensure that edge effects are negligible. Fig. 1 shows an example projection relation for a zone-axis specimen of FCC gold with microscope parameters typical of those used for HAADF-STEM tomography, with example images at selected thicknesses inset. The collection range of the annular detector is defined in terms of its inner and outer angular limits. To investigate how the choice of annular range affects the projection relation, the intensity of the wavefunction arriving in the back focal plane at each scanning position has been azimuthally averaged in 5 mrad annuli. This allows for a variety of detector ranges to be considered by combining the annular bins.

Over the course of an experimental tilt-series acquisition, the specimen is exposed to an extended period of electron beam irradiation. Consequently, this technique is not suitable for beamsensitive materials, or those with any kind of temporal instability.

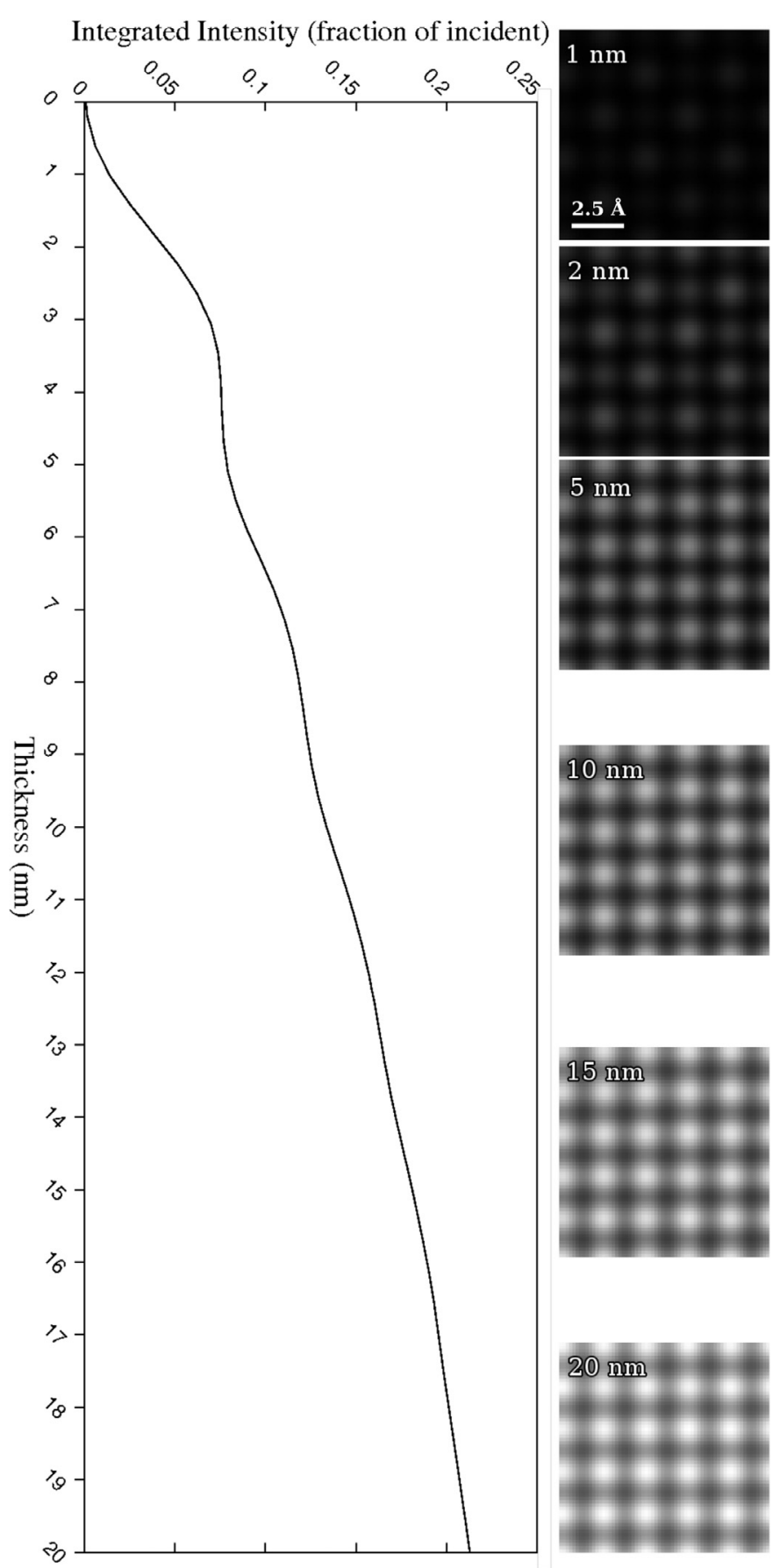

Fig. 1. The relationship between sample thickness and HAADF signal for electron beams propagating through $20 \mathrm{~nm}$ of zone-axis oriented FCC gold. $V_{0}=200 \mathrm{kV}$, $\alpha=10 \mathrm{mrad}, \beta_{i}=100 \mathrm{mrad}, \beta_{0}=250 \mathrm{mrad}$. The inset images show examples of corresponding HAADF images at selected depths with intensities scaled to the dynamic range of the series. The plotted projection relation is the pixel-averaged signal of such images calculated at every slice in the multislice algorithm.

As this work is more applicable to larger specimens with dimensions of the order of tens of nanometers or more, the projection requirement will be assessed in terms of the integrated intensity across all pixels in a $10 \times 10 \AA$ region in the STEM image. The accuracy of atomic resolution tomography, as achieved via atomcounting from zone-axis images, should be considered on a pixelwise or probe integrated crosssection basis, and the conclusions reached in this work are not directly applicable to such techniques $[13,14]$. 
Table 1

\begin{tabular}{lllll}
\multicolumn{6}{l}{ Details of the specimen structures. } \\
\hline Element & Atomic number & Space group & Lattice constants $(\AA)$ & MSD $\left(\AA^{2}\right)$ \\
\hline $\mathrm{C}$ & 6 & Amorphous & - & 0.00182 \\
$\mathrm{Si}$ & 14 & 227 & 5.4309 & 0.006180 \\
$\mathrm{~K}$ & 19 & 229 & 5.328 & 0.1365 \\
$\mathrm{Fe}$ & 26 & 229 & 2.8665 & 0.0071 \\
$\mathrm{Cu}$ & 29 & 225 & 3.6149 & 0.007 \\
$\mathrm{I}$ & 53 & 64 & $7.1802,4.7102,9.8103$ & 0.0046 \\
$\mathrm{Au}$ & 79 & 225 & 4.0782 & 0.0079 \\
$\mathrm{Th}$ & 90 & 225 & 5.0842 & 0.009300 \\
\hline
\end{tabular}

a

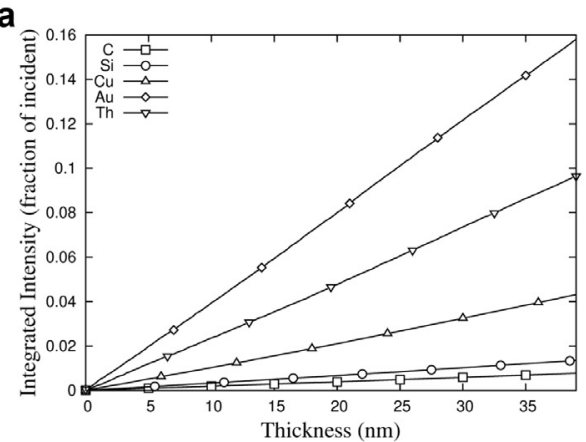

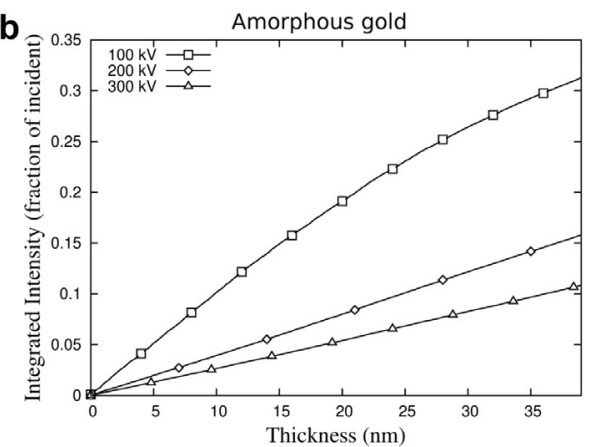

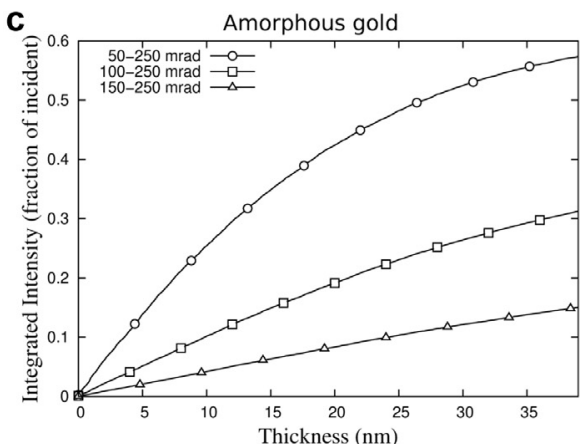

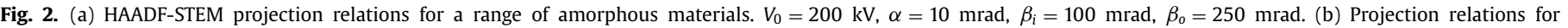

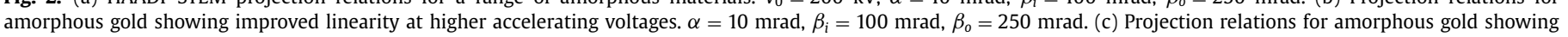
better linearity for greater detector inner angles. $V_{0}=100 \mathrm{kV}, \alpha=10 \mathrm{mrad}$.

The specimen models used in the HAADF-STEM simulations are characterized in terms of the scattering factors of constituent atoms, the geometrical positioning of the atoms, and the meansquare thermal displacements (MSDs) of the atoms. It is not feasible to survey the entire range of possible specimen structures, so a range of chemically homogeneous models in amorphous and crystalline phases have been selected. Table 1 provides an overview of the structural properties of the specimens used in this work. Amorphous materials were produced by randomly generating coordinates within the column volume, with restrictions on the nearest neighbour distance, until the atomic density matched experimentally observed material densities. The scattering factors used in the multislice simulations are those collated by Kirkland in reference [15] and the mean square displacements are those observed at approximately room temperature [16].

\section{Results and discussion}

\subsection{The projection requirement for amorphous materials}

To assess the linearity of HAADF-STEM projections in amorphous materials, multislice simulations have been performed for elemental specimens with a range of atomic numbers. The simulations were performed with an accelerating voltage, $V_{0}$, of $200 \mathrm{kV}$, a convergence angle, $\alpha$, of $10 \mathrm{mrad}$, and a HAADF detector with an inner angle, $\beta_{i}$, of $100 \mathrm{mrad}$, and an outer angle, $\beta_{0}$, of $250 \mathrm{mrad}$. At each slice the intensity of the beam directed towards the HAADF detector has been recorded, and averaged over all scanning beam positions. The resulting average projection relations are shown in Fig. 2a. The HAADF intensities were recorded at each slice, the sparse markers in the projection relation plots are for labelling purposes only and do not represent the densely recorded data points.

Elements with higher atomic numbers have greater projected potentials and, in general, produce a greater occurrence of highangle scattering. This is broadly reflected in the projection relations, although the thorium specimen produces a smaller signal than the gold specimen, despite having a higher atomic number, because the gold atoms are more densely packed. In all cases, the projection relation is approximately linear, and is well suited for tomographic reconstruction. To investigate the effects that varying the accelerating voltage of the electron beam has on the projection relations, two additional simulations have been performed for the amorphous gold specimen with voltages of 100 and $300 \mathrm{kV}$. The resulting projection relations are shown in Fig. 2b. Reducing the accelerating voltage of the beam increases the effective scattering cross-sections, resulting in an increased occurrence of scattering. Consequently, the signal generated for the $100 \mathrm{kV}$ beam is greater than for the 200 and $300 \mathrm{kV}$ beams. However, the greater occurrence of scattering also causes the beam to be more quickly dispersed. The concomitant attenuation of the central beam flux, and the gradual migration of the beam to higher angles due to multiple scattering events, cause a damping of the HAADF signal at greater thicknesses, contravening the projection criterion. This problem can be mitigated by employing larger detector inner angles, which yield projection relations with a gradient that is smaller, but more consistent with increasing specimen thickness, as shown in Fig. 2c. It is concluded that the optimum detector geometry for tilt-series acquisitions of amorphous materials for tomographic reconstruction is given by the largest possible detector range, with the largest inner detector angle for which a useful signal can be measured.

\subsection{The projection requirement for crystalline materials}

Crystalline materials have highly-ordered atomic structures with long-range periodicities. They are anisotropic, with different structures presented along the optical axis as the crystal is rotated. An ideal tilt-series for the purpose of tomographic reconstruction should exhibit a uniform integrated intensity across all orientations, as the total mass-thickness of the specimen remains constant throughout the series. Due to their structural anisotropy, this is not the case for crystalline materials, as demonstrated in Fig. 3, which shows the variation in integrated intensity with crystalline orien- 


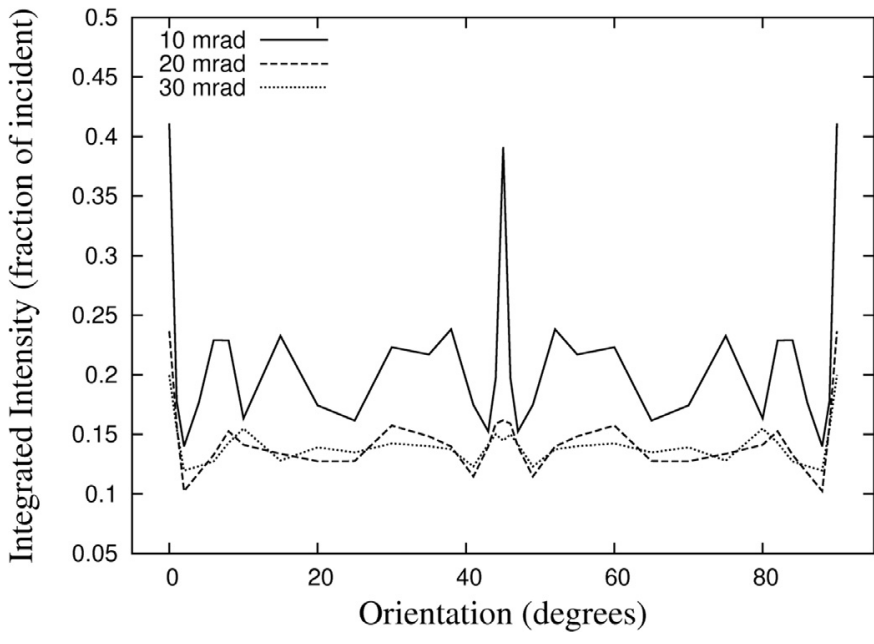

Fig. 3. Variations in the image-integrated HAADF signal for $40 \mathrm{~nm}$ columns of FCC gold with different crystalline orientations. Operating the microscope with larger convergence angles reduces orientation sensitivity. $V_{0}=200 \mathrm{kV}, \beta_{i}=100 \mathrm{mrad}$, $\beta_{0}=250 \mathrm{mrad}$.

tation for $40 \mathrm{~nm}$ columns of gold, at convergence angles of 10, 20, and 30 mrad.

To construct the off-axis models, $60 \times 60 \times 60 \mathrm{~nm}$ cubes of the material, described as a list of atomic coordinates in Cartesian space, were rotated by multiplying the coordinates by a rotation matrix. The specimen column was then isolated by removing atomic coordinates lying outside of a $17 \times 17 \times 40 \mathrm{~nm}$ column located at the centre of the cube. In this manner, the same sample thickness is achieved for zone-axis and off-axis specimens. It should be noted that while the columns have a consistent volume across the series, this does not correspond to a consistent number of atoms, due to the anisotropic crystallography of the material. In this case the sample is rotated about the $<100>$ axis, such that spikes in the integrated intensities occur at orientations of $0^{\circ}$ and $45^{\circ}$ when the $\left[\begin{array}{lll}1 & 0 & 0\end{array}\right]$ and [ $\left[\begin{array}{lll}1 & 1 & 0\end{array}\right]$ crystalline zone-axes are aligned with the optical axis of the microscope such that the scanning electron beam is directed down columns of atoms. The intensity enhancements in these zone-axis images are a consequence of channelling effects which occur when atoms in an aligned column collectively 'draw' the electron beam into the column. This causes an increase in the electron flux impinging on atoms further down the column, and a greater occurrence of high-angle scattering [17]. The channelling-enhanced intensities exhibited in zoneaxis images make them poorly suited for tomographic reconstruction. Scaling of the zone-axis images to match the rest of the tiltseries is inappropriate as the enhancement is not uniformly distributed over the entire image. Images generated using beams with larger convergence angles exhibit less pronounced intensity spikes because they are less susceptible to electron channelling, as they have larger momentum components perpendicular to the optical axis. This effect has previously been observed in experiments reported by Chen et al. [6].

\subsubsection{Imaging along zone-axis orientations}

It has been established that zone-axes images should not, in most cases, be used in conventional tomographic reconstruction algorithms due to their significant intensity enhancement compared with the majority of images, which are off-axis. In this section, the extent to which zone-axis images meet the projection requirement is considered. Fig. 4a shows the projection relations for a number of elemental crystals in zone-axis orientations, the structures of which are given in Table 1 . The simulations were performed for an accelerating voltage of $V_{0}=200 \mathrm{kV}$, a convergence angle, $\alpha=10 \mathrm{mrad}$, and a HAADF detector range of $\beta_{i}=100 \mathrm{mrad}$, $\beta_{0}=250$ mrad. It can be seen that, not only do zone-axis images differ greatly from those of other orientations, but their projection relations are severely non-linear. These projection relations exhibit a similar damping to that seen in the low-voltage projection relation of amorphous gold. In addition, smaller oscillations can be seen, these are caused by the micro-lensing effect of channelling which causes oscillations in the flux of the beam on the axis of atomic columns. The periodicity and magnitude of the oscillations varies with the scattering factors, and inter-atomic spacings present in the specimen. This makes a parametrization of the channelling effect difficult, particularly because strains, faults and vibrational inhomogeneities are prevalent at the nanoscale, and these factors have previously been shown to have a large effect on channelling $[18,19]$.

Fig. $4 \mathrm{~b}$, shows how the choice of the beam convergence angle affects the projection relations for [ $\left[\begin{array}{lll}1 & 0 & 0\end{array}\right]$ zone-axis images of elemental gold. It can be seen that employing greater convergence angles yields a better approximation to a linear projection model as channelling effects are less dominant. A larger convergence angle also produces a tighter focus on the specimen. For an accelerating voltage of $200 \mathrm{kV}$, convergence angles of $10 \mathrm{mrad}, 20 \mathrm{mrad}$, and 30 mrad produce focussed beams with full-width-half-maxima of $1.74 \AA, 0.88 \AA$, and $0.58 \AA$, respectively. However, with a larger convergence angle, the beam diverges more quickly after the focal plane. The depth of field, as defined by Born and Wolf, is proportional to $\lambda / \alpha^{2}$. For the $10 \mathrm{mrad}, 20 \mathrm{mrad}$, and $30 \mathrm{mrad}$ beams at $200 \mathrm{kV}$, this corresponds to depth of field values of $25.1 \mathrm{~nm}$, $6.3 \mathrm{~nm}$, and $2.8 \mathrm{~nm}$. For less-than-atomic resolution tomography, the depth of field is likely the most important factor dictating reconstruction quality, and smaller convergence angles should be chosen.

The choice of accelerating voltage provides an alternative means by which the dominance of channelling effects can be reduced. Fig. 4c, shows projection relations for elemental gold for a range of accelerating voltages. At larger voltages the scattering cross-section is reduced, so multiple scattering is diminished, and channelling is reduced. The cost of improving linearity by increasing the accelerating voltage is that the signal strength is reduced. This makes the optimization of the accelerating voltage difficult for materials containing a large range in atomic numbers, because improving the linearity in regions containing heavier elements compromises the strength of signal generated by regions of lighter elements.

The choice of detector angles has little effect on the extent to which channelling-induced non-linearities occur in the projection relation of HAADF images. However, the angular range in zoneaxis images should be chosen carefully due to the occurrence of longitudinal coherence effects. The coherent scattering from atoms aligned parallel to the optical axis results in a series of interference fringes in the radial distribution, usually in the HAADF range. The position, $\theta_{n}$, of the $n$th maxima is related to the inter-atomic distance along the optical axis, $d$

$\theta_{n}=\arccos \left(1-\frac{n \lambda}{d}\right)$

Where $\lambda$ is the electron wavelength, which varies with accelerating voltage [20]. In the case of crystalline silicon, with a [ $\left.\begin{array}{lll}1 & 0 & 0\end{array}\right]$ axis aligned with the optical axis, for a beam acceleration voltage of $200 \mathrm{kV}(\lambda=2.5 \mathrm{pm})$, the first maxima occurs at $\sim 95 \mathrm{mrad}$. To avoid interference effects, the inner detector angle must be sufficiently low to ensure that the peak corresponding to the first maxima is entirely within the detector range.

Fig. 4d shows the effects of varying the detector geometry on the projection relation for a $200 \mathrm{kV}$ beam with a convergence angle of $\alpha=10$ mrad propagated through an elemental gold column. It can be seen that increasing the inner detector angle from 50 to 
a

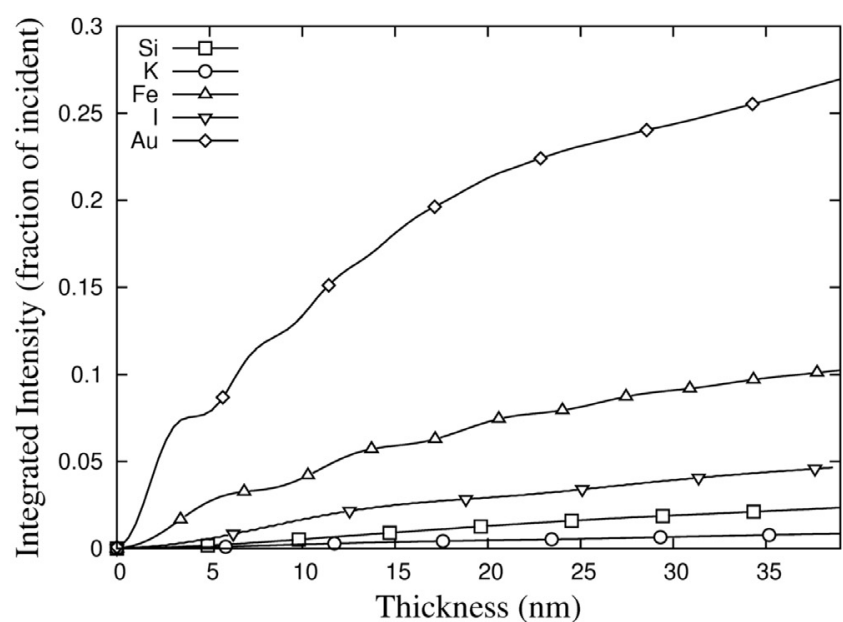

C

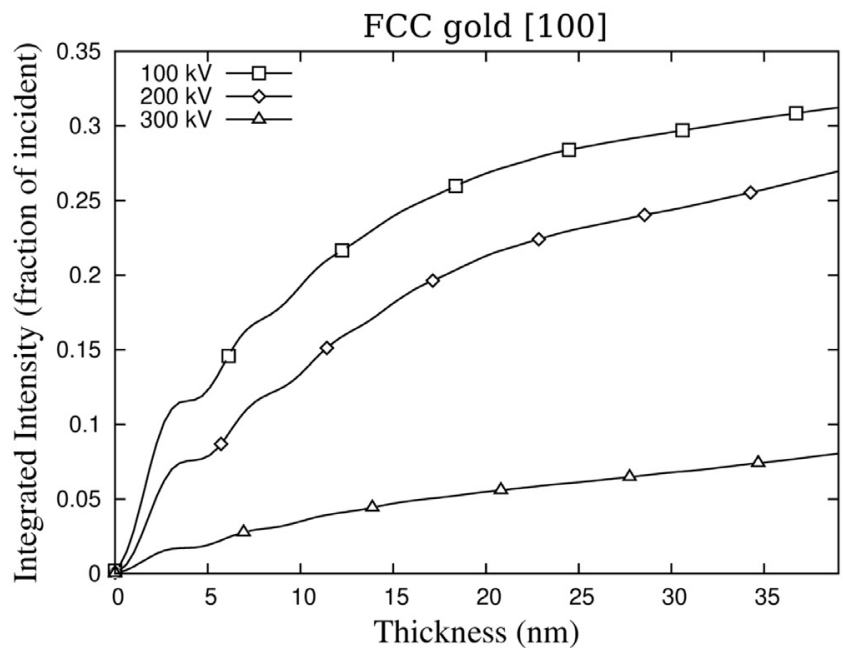

b

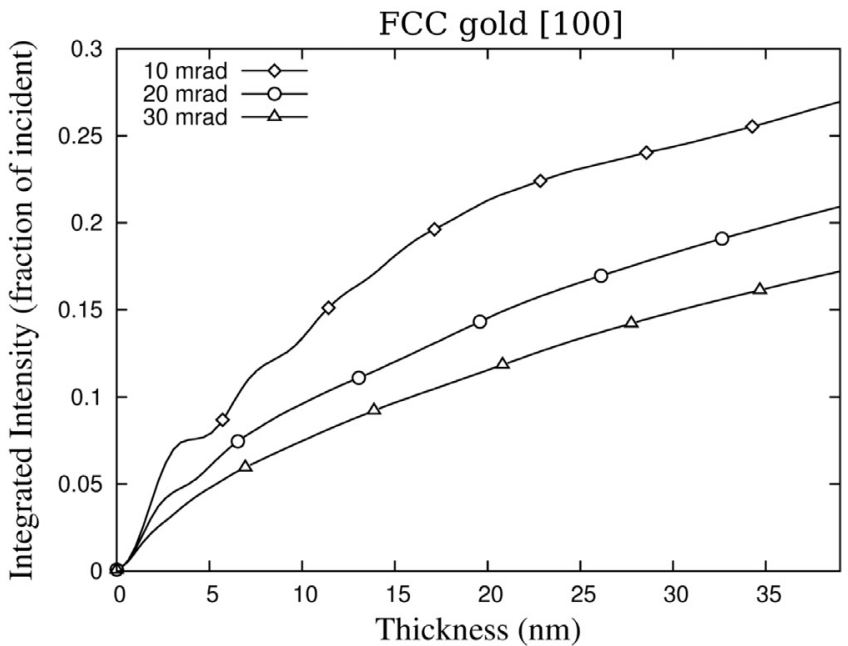

d

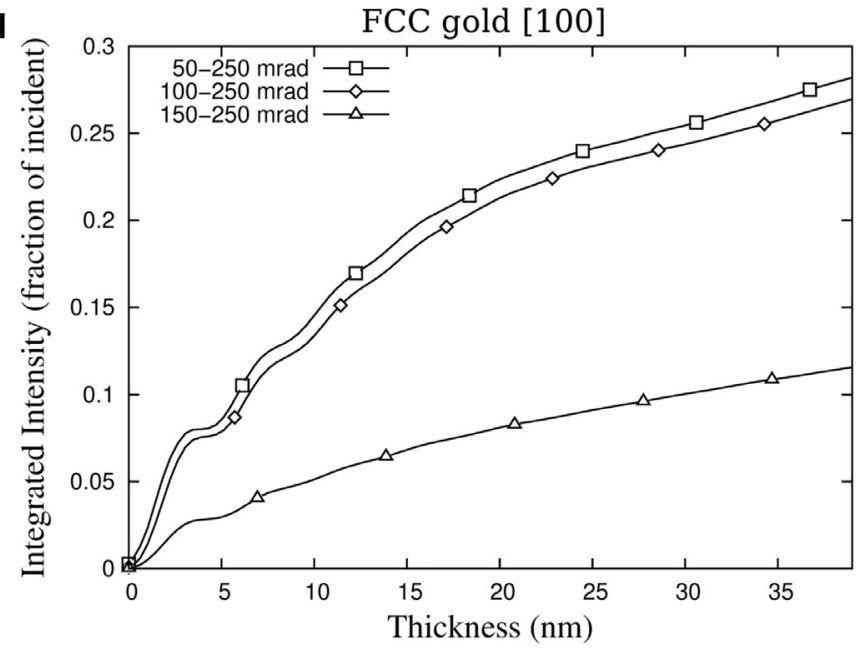

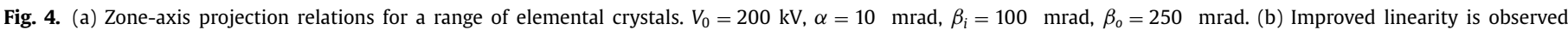

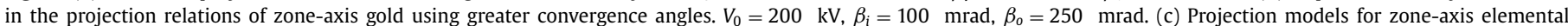

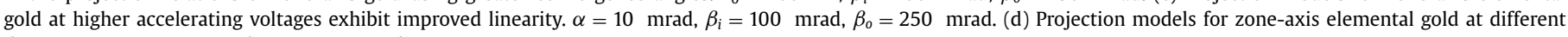
detector ranges. $V_{0}=200 \mathrm{kV}, \alpha=10 \mathrm{mrad}$.

100 mrad has little effect on the linearity of the projection, and results in a small reduction in absolute intensity. Increasing the inner angle to 150 mrad improves the linearity of the projection relation but substantially reduces the absolute intensity by excluding the first maxima of longitudinal coherence.

It has been demonstrated that under common operating conditions, HAADF-STEM images of crystalline specimens in zone-axis orientations are poorly suited for conventional tomographic reconstruction. This is due to both their distinctly enhanced intensities, and their severely non-linear projection relations.

\subsubsection{Imaging along off-axis orientations}

In the previous section it was demonstrated that zone-axis orientations are poorly suited for use in conventional tomographic reconstructions. As a result of this, reconstructions are sometimes performed using only images acquired with the specimen tilted away from zone-axes [21,22]. In this section, the suitability for tomography of off-axis images is investigated with regards to the projection requirement.

It has been shown in Fig. 3 that a $10^{\circ}$ tilt away from a zoneaxis is sufficient to substantially attenuate channelling effects. To investigate off-axis projection relations, simulations have been performed for a variety of crystalline materials with a $10^{\circ}$ tilt away from a zone-axis, for an accelerating voltage of $V_{0}=200 \mathrm{kV}$, a convergence angle of $\alpha=10 \mathrm{mrad}$, and a HAADF detector range of $\beta_{i}=100 \mathrm{mrad}, \beta_{0}=250 \mathrm{mrad}$. In performing these multislice simulations of off-axis specimens, the slice thickness has been adjusted to best match the new periodicity along the optical axis. The resulting projection relations are shown in Fig. 5a. These projection relations exhibit better behaviour for reconstruction than the zoneaxis projections due to their more consistent gradients, in contrast to the oscillatory gradients seen in Fig. 4. The off-axis projections models do, however, exhibit a similar long-range damping effect to that observed in amorphous materials at lower voltages.

As these off-axis images are less dominated by channelling effects, the convergence angle of the electron beam has relatively little effect on the form of the projection relation. This is shown in Fig. 5b, in which projection relations are plotted for a beam propagating through off-axis elemental gold with an accelerating voltage of $V_{0}=200 \mathrm{kV}$, a HAADF detector range of $\beta_{i}=100 \mathrm{mrad}, \beta_{0}=$ 250 mrad, and beam convergence angles of 10,20 , and 30 mrad. As the convergence angle does not substantially affect the projection linearity, the larger depth of field associated with smaller convergence angles is expected to yield the most accurate reconstructions.

As in the zone-axis and amorphous cases, the damping of the HAADF signal at greater thicknesses in the specimen can be re- 
a

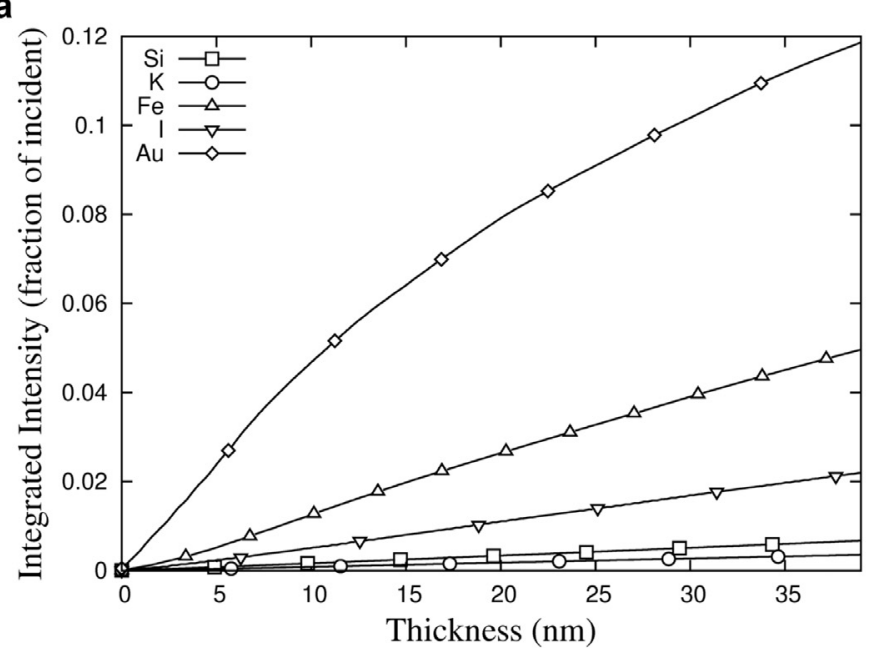

c

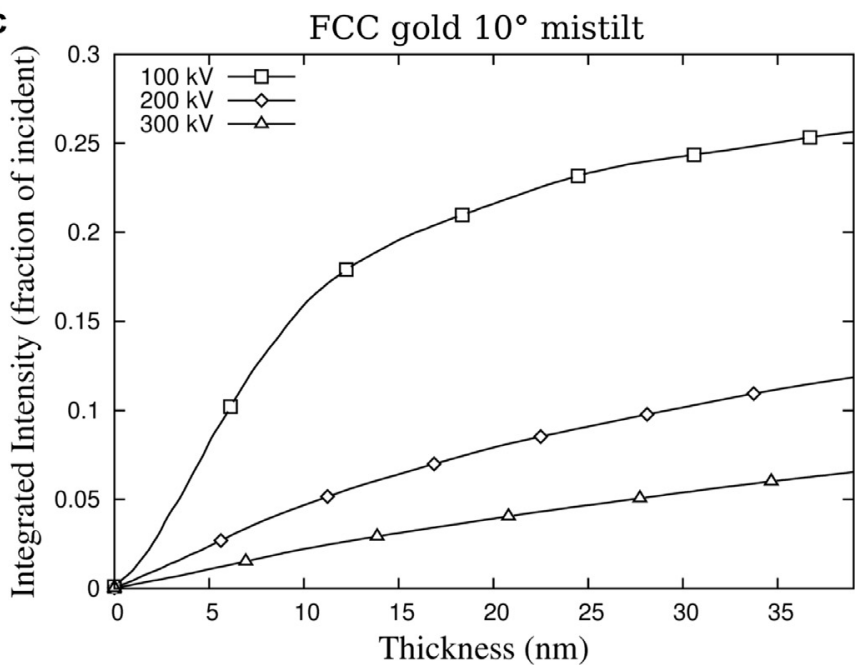

b

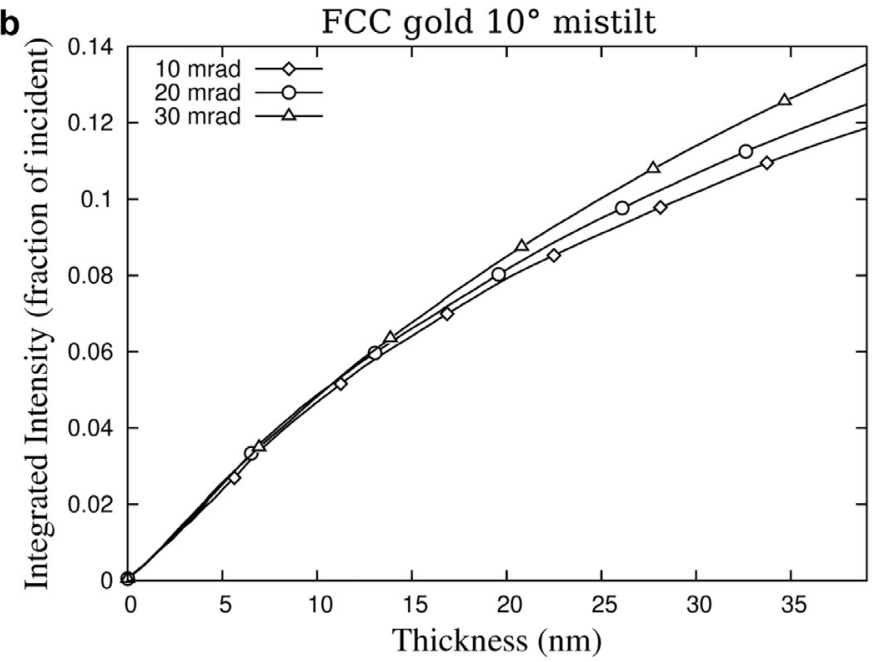

d

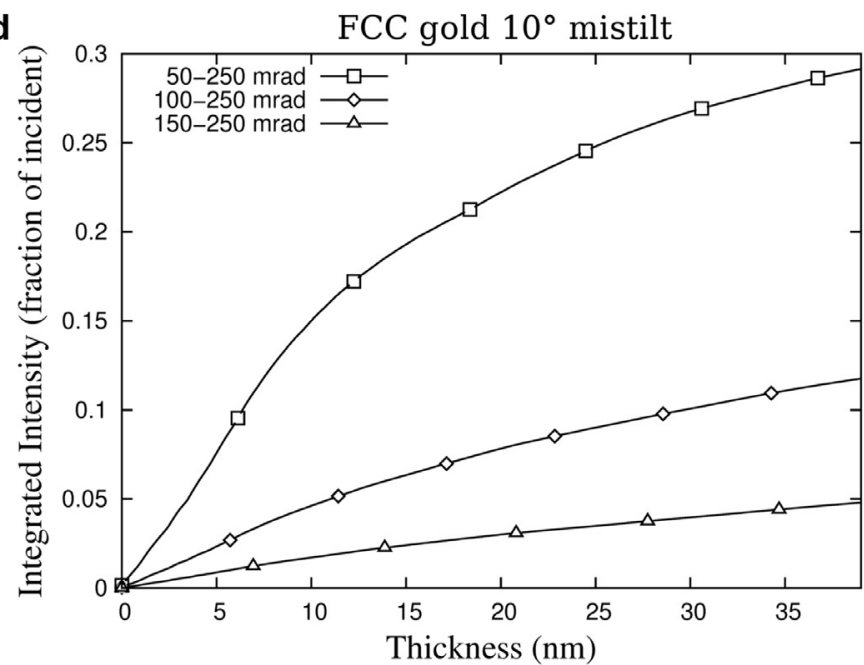

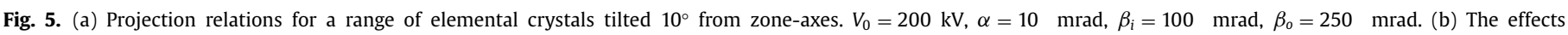

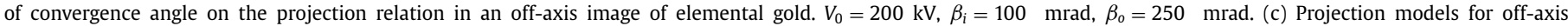

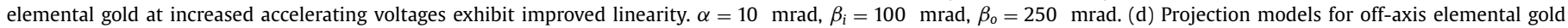
at different detector ranges. $V_{0}=200 \mathrm{kV}, \alpha=10 \mathrm{mrad}$.

duced by increasing the accelerating voltage. Fig. $5 \mathrm{c}$ demonstrates this for the case of elemental gold tilted $10^{\circ}$ from the $\left[\begin{array}{lll}1 & 0 & 0\end{array}\right]$ zone axis, with a convergence angle of 10 mrad, a HAADF detector range of $\beta_{i}=100 \mathrm{mrad}, \beta_{0}=250 \mathrm{mrad}$, and accelerating voltages of 100,200 , and $300 \mathrm{kV}$. As in previous cases, the accelerating voltage should be selected as a compromise between signal strength and projection linearity, with the smallest useful signal strength giving the best linearity.

Unlike the zone-axis case, off-axis images are not significantly susceptible to longitudinal coherence effects, consequently, no consideration need be made for interference fringes when selecting HAADF detector ranges. Fig. $5 \mathrm{~d}$ shows the projection relations for off-axis elemental gold using an electron beam with an accelerating voltage of $V_{0}=200 \mathrm{kV}$, a convergence angle of $10 \mathrm{mrad}$, and HAADF detector ranges of 50-250, 100-250, and 150-250 mrad. It can be seen that the damping of intensities at greater thicknesses can be reduced by increasing the inner detector angle, this effect has recently been observed experimentally[23]. The intensity distribution of the electron wavefunction falls away quickly at higher angles, consequently, improving the linearity of the projection relation by increasing the inner detector angle incurs a substantial loss in signal strength.
In zone-axis HAADF-STEM images of materials containing more than one element, columnar intensities have been found to vary depending on the arrangement of the elements along the beam axis [24-26]. This can be attributed to the oscillating on-axis beam flux caused by electron channelling. Similarly, it is expected that the different rates of beam dispersion and signal damping observed here for different materials may cause chemical ordering sensitivity in absolute intensity measurements.

Many nano-scale materials of technological interest take a crystalline phase, often with twinning to minimise surface energy effects. It is clearly desirable that such materials can be characterized in three dimensions using tilt-series STEM tomography. In all the cases considered for this work, the off-axis images meet the minimum requirement of tilt-series transmission tomography, as they exhibit a monotonic relationship between mass-thickness and signal intensity. It is thus concluded that HAADF-STEM images of electron-transparent specimen at orientations away from zone-axes are suitable for tilt-series tomographic reconstruction. However, such images do not generally follow the linear model assumed by conventional reconstruction algorithms.

Simulations offer a means of estimating the extent to which non-linearities are likely to occur for a given specimen, and could 
be used to inform the correction of reconstructions. However, the difficulty in quantifying all the necessary experimental parameters, and the computational expense of the rigorous simulations necessary to reproduce multiple scattering phenomena in non-periodic specimen are substantial, and place a significant time constraint on such approaches.

\section{Conclusion}

Multislice simulations have been performed to investigate the extent to which HAADF-STEM meets the projection requirement for tilt-series tomographic reconstruction. A range of specimens have been considered, and the microscope parameter space has been surveyed to inform the optimization of experimental set-up for tilt-series acquisitions.

Imaging of amorphous materials has been found to give a close approximation to a linear projection model in most cases. For heavier elements, multiple scattering can redirect the beam away from the HAADF detector, causing a damping of the HAADF signal at greater thicknesses. This effect can be reduced by employing increased accelerating voltages and larger inner angles of the annular detector, but the measured signal strength will be compromised.

Images of crystalline materials acquired along zone-axis orientations were found to be poorly suited for conventional tomographic reconstruction due to channelling effects which cause intensity mismatches compared to the rest of the tilt-series, and projection relations with oscillatory gradients.

Images of crystalline materials tilted away from zone-axis orientations, are better suited to use in tomographic reconstruction, however, they are still susceptible to non-linearities. The form of the non-linearity will vary with the crystalline structure, chemical composition, and orientation of the specimen, making accurate correction of the non-linearity difficult. Better approximations to linear behaviour can be achieved by operating the microscope with higher accelerating voltages and greater detector inner angles.

For specimens containing multiple elements with a large range in atomic numbers, linearity through thick regions of heavy elements must be compromised in order to maintain a sufficient signal strength in thin regions of lighter elements. This irreconcilable scenario provides a strong impetus for the development of novel non-linear reconstruction procedures.

\section{Acknowledgements}

This research is supported by the Dutch Technology Foundation STW (http://www.stw.nl/), which is part of the Netherlands Organization for Scientific Research (NWO), which is partly funded by the Ministry of Economic Affairs, Agriculture and Innovation under number 13314. This work is part of the research programme with project numbers SH-331-15 and 639.073.506, which are (partly) financed by the Netherlands Organisation for Scientific Research (NWO).

\section{References}

[1] P. Midgley, M. Weyland, 3D electron microscopy in the physical sciences: the development of Z-contrast and EFTEM tomography, Ultramicroscopy 96 (34) (2003) 413-431. Proceedings of the International Workshop on Strategies and Advances in Atomic Level Spectroscopy and Analysis.

[2] C. Kübel, A. Voigt, R. Schoenmakers, M. Otten, D. Su, T.-C. Lee, A. Carlsson, J. Bradley, Recent advances in electron tomography: TEM and HAADF-STEM tomography for materials science and semiconductor applications, in: Microscopy and Microanalysis : the Official Journal of Microscopy Society of America, Microbeam Analysis Society, Microscopical Society of Canada 11, 2005, pp. 378-400.

[3] S. Bals, G.V. Tendeloo, C. Kisielowski, A new approach for electron tomography: annular dark-field transmission electron microscopy, Adv. Mater. 18 (2006) $892-895$.

[4] J.M. Lebeau, S.D. Findlay, L.J. Allen, S. Stemmer, Quantitative atomic resolution scanning transmission electron microscopy, Phys. Rev. Lett. 100 (May) (2008) $1-4$

[5] W.V.D. Broek, A. Rosenauer, B. Goris, G.T. Martinez, S. Bals, S.V. Aert, D.V. Dyck, Correction of non-linear thickness effects in HAADF STEM electron tomography, Ultramicroscopy 116 (2012) 8-12.

[6] Z. Chen, M. Weyland, X. Sang, W. Xu, J. Dycus, J. LeBeau, a.J. D’Alfonso, L. Allen, S. Findlay, Quantitative atomic resolution elemental mapping via absolute-scale energy dispersive x-ray spectroscopy, Ultramicroscopy 168 (2016) 7-16.

[7] S.J. Pennycook, D.E. Jesson, High-resolution z-contrast imaging of crystals, Ultramicroscopy 37 (1991) 14-38.

[8] Z. Saghi, X. Xu, G. Möbus, Electron tomography of regularly shaped nanostructures under non-linear image acquisition, J. Microsc. 232 (2008) 186-195.

[9] X. Xu, Z. Saghi, R. Gay, G. Möbus, Reconstruction of 3d morphology of polyhedral nanoparticles, Nanotechnology 18 (2007) 225501.

[10] G.T. Herman, Correction for beam hardening in computed tomography., Phys. Med. Biol. 24 (1979) 81-106.

[11] J.M. Lebeau, S.D. Findlay, L.J. Allen, S. Stemmer, Standardless atom counting in scanning transmission electron microscopy, Nano Lett. 10 (4405) (2010) 4405-4408

[12] R. Aveyard, B. Rieger, Tilt series STEM simulation of a $25 \times 25 \times 25 \mathrm{~nm}$ semiconductor with characteristic x-ray emission, Ultramicroscopy 171 (2016) 96-103.

[13] S.V. Aert, K.J. Batenburg, M.D. Rossell, R. Erni, G.V. Tendeloo, Three-dimensional atomic imaging of crystalline nanoparticles., Nature 470 (7334) (2011) $374-377$.

[14] H. E., K.E. MacArthur, T.J. Pennycook, E. Okunishi, a.J. D’Alfonso, N.R. Lugg, L.J. Allen, P.D. Nellist, Probe integrated scattering cross sections in the analysis of atomic resolution HAADF STEM images, Ultramicroscopy 133 (2013) 109-119

[15] E.J. Kirkland, Advanced Computing in Electron Microscopy, second ed., Springer, 2010

[16] L.-M. Peng, G. Ren, S.L. Dudarev, M.J. Whelan, Debye-waller factors and absorptive scattering factors of elemental crystals, Acta Crystallogr. Sect. A Found. Crystallogr. 52 (1996) 456-470.

[17] D.V. Dyck, M.O. De Beeck, A simple intuitive theory for electron diffraction, Ultramicroscopy 64 (1996) 99-107.

[18] R. Aveyard, R. Ferrando, R.L. Johnston, J. Yuan, Modeling nanoscale inhomogeneities for quantitative HAADF STEM imaging, Phys. Rev. Lett. 113 (7) (2014) 075501.

[19] V. Grillo, The effect of surface strain relaxation on HAADF imaging, Ultramicroscopy 109 (12) (2009) 1453-1464.

[20] D.E. Jesson, S.J. Pennycook, Incoherent imaging of crystals using thermally scattered electrons, Proc. R. Soc. A 449 (1936) (1995) 273-293.

[21] M. Scott, C. Chen, M. Mechlenburg, C. Zhu, R. Xu, C. Regan, J. Miao, P. Ercius, U. Dahmen, Electron tomography at $2.4 \AA$ resolution, Microsc. Microanal. 18 (2012) 522-523.

[22] W. Van den Broek, S. Van Aert, D. Van Dyck, A model based atomic resolution tomographic algorithm, Ultramicroscopy 109 (12) (2009) 1485-1490.

[23] M. Niehle, Electron tomography and microscopy on semiconductor heterostructures, Humboldt-Universität zu Berlin, Mathematisch-Naturwissenschaftliche Fakultät, 2016 Ph.d. thesis.

[24] B. Bollig, H.-G. Fischer, E. Kubalek, Multislice simulation of high-resolution scanning transmission electron microscopy z-contrast images of semiconductor heterointerfaces, Scanning 18 (4) (1996) 291-300.

[25] E. Rotunno, M. Albrecht, T. Markurt, T. Remmele, V. Grillo, Three dimensional analysis of the composition in solid alloys by variable probe in scanning transmission electron microscopy, Ultramicroscopy 146 (2014) 62-70.

[26] B. Esser, A. Hauser, R. Williams, L. Allen, P. Woodward, F. Yang, D. McComb, Quantitative stem imaging of order-disorder phenomena in double perovskite thin films, Phys. Rev. Lett. 117 (17) (2016) 176101. 